CORRECTION

https://doi.org/10.1038/s41586-018-0635-8

\section{Author Correction: A multi-cohort study of the immune factors associated with $M$. tuberculosis infection outcomes}

Roshni Roy Chowdhury, Francesco Vallania, Qianting Yang, Cesar Joel Lopez Angel, Fatoumatta Darboe, Adam Penn-Nicholson, Virginie Rozot, Elisa Nemes, Stephanus T. Malherbe, Katharina Ronacher, Gerhard Walzl, Willem Hanekom, Mark M. Davis, Jill Winter, Xinchun Chen, Thomas J. Scriba, Purvesh Khatri \& Yueh-hsiu Chien

Correction to: Nature https://doi.org/10.1038/s41586-018-0439-x, published online 22 August 2018.

In this Letter, data from 2 out of the 17 progressors were inadvertently binned into the wrong time intervals. We have now re-analysed the entire dataset with all data points binned correctly with respect to the time intervals before tuberculosis (TB) diagnosis, and the first graph in Fig. $4 \mathrm{~b}$ has been corrected (see Fig. 1 of this Amendment for the original figure). In the revised analysis, the time intervals are set at: $>400$, 201-400, 101-200 and 0-100 days before TB diagnosis (intervals in the original Fig. $4 \mathrm{~b}$ were: $361-540,181-360,61-180$ and $0-60$ days). The comparisons between the levels of natural killer (NK) cells 1 year before TB diagnosis and at TB diagnosis, and between approximately 7 months before TB diagnosis and at TB diagnosis remain statistically significant $(P=0.0312$ and $P=0.0039$, respectively; $P=0.0078$ and $P=0.0156$ in the original Letter). The original observation of a

Original Fig. 4b
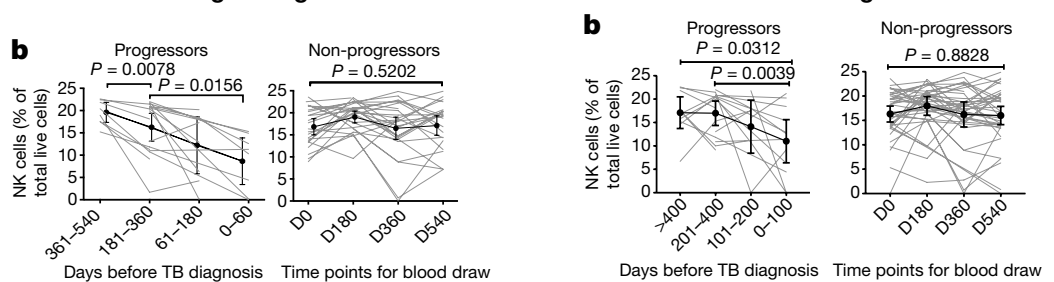

Original Fig. 4c

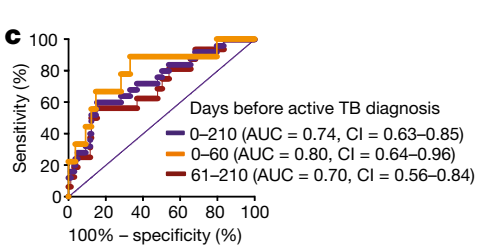

significant decrease in the percentage of NK cells during the progression to active TB remains unchanged.

In the original Letter, all non-progressors that had measurements for at least three time points were included. We have now included all non-progressors that had longitudinal measurements for at least two time points $(n=41)$. The second graph in Fig. $4 \mathrm{~b}$ and the Reporting Summary have been corrected (see Fig. 1 of this Amendment for the original figure, and Supplementary Information of this Amendment for the original Reporting Summary). The revised analysis shows no significant change in the frequency of NK cells $(P=0.8828 ; P=0.5202$ in the original Letter) during the two-year study period.

The receiver operating characteristic curves in Fig. $4 \mathrm{c}$ have been corrected (see Fig. 1 of this Amendment for the original figure). The new analysis shows an area under the curve (AUC) of 0.74 (95\% confidence interval (CI) $0.58-0.90$ ) in the three months (100 days) before active TB diagnosis ( $\mathrm{AUC}=0.80, \mathrm{CI}=0.64-0.96$ in the two months (60 days) before active TB diagnosis in the original Letter), and an AUC of $0.69(\mathrm{CI}=0.57-0.82)$ in the seven months (200 days) before active TB diagnosis ( $\mathrm{AUC}=0.74$; $\mathrm{CI}=0.63-0.85$ in the 210 days before active TB diagnosis in the original Letter). These analyses do not change any of our conclusions.

In addition, the name of author Qiantian Yang should have been spelled Qianting Yang, and the affiliation of author Stephanus T. Malherbe should only be 'Department of Science and Technology, National Research Foundation Centre of Excellence for Biomedical Tuberculosis Research, Stellenbosch University, Stellenbosch, South Africa', and should not include 'Catalysis Foundation for Health, Emeryville, CA, USA', as was originally listed. The Letter has been corrected online.

Supplementary information is available in the online version of this Amendment.

Fig. 1 | This figure displays the corrected panels and the incorrect published panels from Fig. $4 \mathrm{~b}, \mathrm{c}$ of the original Letter. 